Gynäkologe 2006 ·39:501-501 DOI 10.1007/s00129-006-1868-4 Online publiziert: 11. Juli 2006

๑) Springer Medizin Verlag 2006

\author{
L. Beck $\cdot$ H. Hepp ${ }^{2}$ \\ ${ }^{1}$ Düsseldorf \\ ${ }^{2}$ Buch am Ammersee
}

\title{
Zum Geburtstag von Volker Friedberg
}

dem Gebiet der Geburtshilfe, insbesondere der Untersuchungen zum Schwangerschaftshochdruck, zur Eklampsie und Präeklampsie - in Zusammenarbeit mit internistischen Forschergruppen. Gemeinsame Tagungen mit Pädiatern in Bad Schachen auf Einladung von Friedberg und dem Pädiater Ewerbeck haben bereits in den 6oerJahren zu einer engen Zusammenarbeit im Sinne der sich später entwickelnden Perinatologie geführt und das Gebiet der Neonatologie gefördert.

\section{( ) Er führte die Senologie in die Frauenheilkunde zurück}

Schwerpunkt seiner klinischen Tätigkeit war die operative Gynäkologie mit kontinuierlicher Fortentwicklung der gynäkologischen Onkochirurgie. Während seiner Ausbildung bei Kräuter in Mainz (Arzt für Gynäkologie und Chirurgie) erlernte Friedberg die operative Therapie des Brustkrebses und war wohl der erste Hochschullehrer und Klinikdirektor in der BRD, der die klinische und operative Senologie in die Frauenheilkunde zurückgeführt hat.

Exenterationen bei ausgedehnten gynäkologischen Karzinomen wurden in Zusammenarbeit mit der Mainzer Urologischen Klinik durchgeführt, wobei die vom Krebs befallene Gebärmutter und - falls erforderlich - Harnblase und untere Darmabschnitte entfernt wurden. Auch das gesamte Spektrum der rekonstruktiven Chirurgie wurde in der Mainzer Frauenklinik beherrscht.

Sehr früh hat Friedberg nach einem Besuch bei dem Internisten Kalk in Kassel die Laparoskopie in die gynäkologische Dia- gnostik übernommen - auch wenn zu jener Zeit die technischen Voraussetzungen noch mangelhaft waren.

Es ist das Verdienst von Volker Friedberg, dass zahlreiche seiner Schüler Chefärzte und Leitende Ärzte an Univ.-Frauenkliniken geworden sind (Beck, Friese, Hepp, Höckel, Kreienberg, Knappstein, Melchert). Wir denken mit Dankbarkeit zurück an die Jahre gemeinsamer erfolgreicher Arbeit in einer offenen, inspirierenden Atmosphäre und danken für die in Vertrauen und gegenseitigem Respekt gewährte Unterstützung.

Zahlreiche ehrenvolle Aufgaben und Ehrungen wurden Volker Friedberg zuteil; er war Präsident der Deutschen Gesellschaft für Gynäkologie und Geburtshilfe (19721974). Er wurde deren Ehrenmitglied und erhielt die Karl-Kaufmann-Plakette - die höchste wissenschaftliche Auszeichnung der Gesellschaft. Zahlreiche Anfragen und Berufungen während der Zeit seiner Tätigkeit in Saarbrücken und Mainz haben ihn erreicht (Berlin, Marburg, Bonn, Heidelberg, Freiburg, Zürich).

Seine Familie und seine ehemaligen Schüler waren froh, dass Volker Friedberg nach Mainz zurückgekehrt und dort bis zu seiner Emeritierung geblieben ist.

Glück und Wohlergehen in der Heimat am Bodensee - ad multos annos! 